\title{
EDITORIAL \\ Potential survival benefit of resection in butterfly glioblastoma
}

\author{
Howard Colman, MD, PhD \\ Huntsman Cancer Institute and Department of Neurosurgery, University of Utah, Salt Lake City, Utah
}

$\mathrm{T}$ IHE combination of butterfly involvement of both hemispheres of the brain and pathological diagnosis of glioblastoma (GBM) is a well-known phenomenon to clinicians involved in the care of GBM patients. As described by many investigators over the years, disease prognosis in these patients is unfortunately quite poor. ${ }^{2,4,5}$ This poor prognosis leads to a therapeutic dilemma for both patients and clinicians: should one be aggressive with neurosurgical resection and other adjuvant treatments to achieve better disease control, or do the potential toxicities and decreased quality of life associated with more aggressive treatment outweigh the relatively low likelihood of a durable benefit in the majority of patients? Thus, determining what are the major prognostic factors and what interventions have the highest likelihood of significant benefit in selected subcategories of patients is crucial.

A number of prior studies have examined some of these questions. Unfortunately, given the relatively small patient population and the lack of prospective trials, there are relatively few publications with sufficient patient numbers to determine the key prognostic factors and relative benefits of various therapeutic interventions. Nonetheless, there have been several important observations from prior retrospective analyses and larger case series. ${ }^{2,4,5}$ Several of these studies have identified prognostic factors for butterfly GBM (bGBM) that are similar to those for other GBMs including patient age and performance status. For bGBM specifically, the volume of the initial tumor and receiving adjuvant treatment with radiation and/or chemotherapy have been identified as prognostic factors in some studies. $2,4,5$

On the question of the optimal neurosurgical approach for bGBM, several studies have highlighted approaches for maximizing extent of resection (EOR) while minimizing the risk of potentially devastating neurological deficits such as abulia and akinetic mutism. ${ }^{1,2,4,5}$ One of the major findings from earlier case series is that more extensive resection is feasible with reasonable surgical risks, at least in some patients with bGBM. All of these studies also found a benefit of resection versus biopsy $y^{2,4,5}$ and/or a benefit of an increased percentage of resection on overall survival. These benefits appear clinically significant, with a median survival range of 1.5-3.5 months with biopsy and 7.5-8.8 months with resection. ${ }^{2,4,5}$ However, these observations suffer from a typical limitation of retrospective analyses of two different treatment approaches, namely that those treatment decisions were based on a clinical assessment at the time, and thus could be (and probably are) biased by differences in the biopsy versus resection patient populations.

It is in this context that the study by Dayani et al. ${ }^{3}$ attempts to further clarify the safety and outcomes of resection in bGBM. These authors screened an impressive number of GBMs (1746 cases) from their institutional database to identify 42 cases that met the criteria for bGBM based on enhancing tumor involving both hemispheres and the corpus callosum. Consistent with data in prior publications, overall survival for the entire group with bGBM was poor, with a median survival of 3.2 months from diagnosis and an overall 6-month survival rate of $38.1 \%$. Age, Karnofsky Performance Status (KPS) at diagnosis, preoperative tumor volume, and EOR were found to be significant prognostic factors for overall survival in the univariate analysis. Extent of resection was found to confer a better prognosis than biopsy (HR 0.37, $\mathrm{p}=0.009$ ) with a minimum EOR of $86 \%$ required to observe survival benefits (HR 0.054, $\mathrm{p}=0.03$ ). One interesting observation in their study that goes beyond prior analyses is that the EOR specifically of the corpus callosum component of the tumor was not significantly associated with overall survival. 
Another important observation in their study is that the benefits of EOR, in terms of both median and landmark survival, appear to be clinically meaningful. If more aggressive resection simply resulted in a modest increase of a few months in median survival, this could be counterbalanced by the increased incidence of immediate and late neurological deficits, as well as the increased rehabilitation time that results. In the current study, however, median survival of the patients undergoing resection was 14.06 months compared to only 2.53 months for those undergoing biopsy only (HR 0.37, p = 0.009), which is a larger difference than that observed in prior studies. The landmark analyses also demonstrated the benefits of greater resection at multiple time points, with survival of $71.4 \%$ versus $20 \%$ at 6 months, $57.1 \%$ versus $8 \%$ at 12 months, and $21.4 \%$ versus $4 \%$ at 18 months for resection versus biopsy, respectively. One of the long-standing concerns that has historically biased neurosurgeons against more aggressive resection in this patient population is the risk of immediate and persistent neurological deficits. While the current study did note a $35.7 \%$ rate of immediate postoperative deficits after resection, only $7.14 \%$ of the deficits were persistent. There was also an average decrease of 10 points in the KPS score after surgery for patients undergoing resection, but this finding was not statistically significant. One concern about these analyses, as described above, is that differences in the baseline characteristics of patients who underwent resection versus biopsy were observed. Specifically, patients undergoing resection in the current study were younger and had a better preoperative KPS. In addition, while resection patients did not have a smaller overall tumor volume, they did have a lower percentage of tumor volume on the dominant side. Despite these imbalances, however, multivariate analysis demonstrated that preoperative tumor volume and surgical approach (resection vs biopsy) remained independent prognostic factors after accounting for patient-specific characteristics.

The paper by Dayani et al. ${ }^{3}$ confirms a number of findings from prior studies regarding the role of more extensive surgery in bGBM and successfully characterizes a number of the important clinical and prognostic factors in this population. The study further demonstrates that the consideration of more aggressive resection may be reasonable in a larger percentage of patients with bGBM than previously appreciated. However, it also remains clear that the overall prognosis for many of these patients is still poor and that patients with negative prognostic factors and in whom extensive resection ( $>86 \%$ in the current study) is not possible have a life expectancy that is measured in months. Thus, it appears that careful selection of patients in whom to be more aggressive in terms of neurosurgical resection remains very important. While the study cannot directly answer these questions based on its retrospective and non-controlled nature, several important factors that may identify appropriate patients for more aggressive surgery (and adjuvant treatment) include a younger age, better KPS score, lower percentage of tumor involvement on the dominant side, smaller presurgical volume, and preoperative assessment indicating that $>86 \%$ resection is achievable. Additional studies with independent data sets, including those with more extensive molecular characterization of the tumors, are necessary to validate the findings of the current study, to determine the association between resection benefits and molecular phenotype, and to build a better multivariate model of those patients with bGBM who are the best candidates for aggressive neurosurgical resection and adjuvant treatment.

https://thejns.org/doi/abs/10.3171/2018.3.FOCUS18152

\section{References}

1. Burks JD, Bonney PA, Conner AK, Glenn CA, Briggs RG, Battiste JD, et al: A method for safely resecting anterior butterfly gliomas: the surgical anatomy of the default mode network and the relevance of its preservation. J Neurosurg 126:1795-1811, 2017

2. Chaichana KL, Jusue-Torres I, Lemos AM, Gokaslan A, Cabrera-Aldana EE, Ashary A, et al: The butterfly effect on glioblastoma: is volumetric extent of resection more effective than biopsy for these tumors? J Neurooncol 120:625-634, 2014

3. Dayani F, Young JS, Bonte A, Chang EF, Theodosopoulos P, McDermott MW, et al: Safety and outcomes of resection of butterfly glioblastoma. Neurosurg Focus 44(6):E4, 2018

4. Dziurzynski K, Blas-Boria D, Suki D, Cahill DP, Prabhu SS, Puduvalli V, et al: Butterfly glioblastomas: a retrospective review and qualitative assessment of outcomes. J Neurooncol 109:555-563, 2012

5. Opoku-Darko M, Amuah JE, Kelly JJP: Surgical resection of anterior and posterior butterfly glioblastoma. World Neurosurg 110:e612-e620, 2018

\section{Disclosures}

Dr. Colman has been a consultant for Roche, Genentech, Novocure, Omniox, Insys, and Abbvie; has participated in an educational symposium for Merck; and has clinical research support in the form of institutional contracts from Newlink Genetics, Plexxikon, Kadmon, Orbus, Merck, DNATrix, Abbvie, and Beigene for the conduct of clinical trials. 